\title{
Exploiting the Hamiltonian structure of a neural field model
}

\author{
A.J. Elvin ${ }^{\mathrm{a}, *}$, C.R. Laing ${ }^{\mathrm{b}}$, R.I. McLachlan ${ }^{\text {c }}$, M.G. Roberts ${ }^{\mathrm{b}}$ \\ a Department of Mathematics, The University of Auckland, Private Bag 92019, Auckland, New Zealand \\ ${ }^{\mathrm{b}}$ Institute of Information and Mathematical Sciences, Massey University, Private Bag 102904 NSMC, Auckland, New Zealand \\ ${ }^{\mathrm{c}}$ Institute of Fundamental Sciences, Massey University, Private Bag 11-222, Palmerston North, New Zealand
}

\section{A R T I C L E I N F O}

\section{Article history:}

Available online xxxx

Communicated by J. Lega

\section{PACS:}

87.10.-e

87.10.Ed

89.75.Kd

\section{Keywords:}

Pattern formation

Hamiltonian

Bifurcation

Homoclinic

\begin{abstract}
A B S T R A C T
We study the unexpected disappearance of stable homoclinic orbits in regions of parameter space in a neural field model with one spatial dimension. The usual approach of using numerical continuation techniques and local bifurcation theory is insufficient to explain the qualitative change in the model's behaviour. The lack of robustness of the model to small perturbations in parameters is surprising, and the phenomenon may be of broader significance than just our model. By exploiting the Hamiltonian structure of the time-independent system, we develop a numerical technique with which we discover that a small, separate solution curve exists for a range of parameter values. As the firing rate function steepens, the small curve causes the main curve to break and stable homoclinic orbits are destroyed in a region of parameter space. Numerically, we use level set analysis to find that a codimension-one heteroclinic bifurcation occurs at the terminating ends of the solution curves. By replacing the firing rate function with a step function, we show analytically that the bifurcation is related to the value of the firing threshold. We also show the existence of heteroclinic orbits at the breakpoints using a travelling front analysis in the time-dependent system.
\end{abstract}

(C) 2009 Elsevier B.V. All rights reserved.

\section{Introduction}

Pattern formation in spatially extended systems is an area of study that has shown major progress within the last few decades. Systems from a wide range of biological, geophysical, ecological, physical and material sciences are studied, making pattern formation an interdisciplinary science. Spatial patterns can be stationary, travelling or disordered in both space and time, i.e. spatio-temporally chaotic. Spatially localised solutions are of importance in many different areas, such as the study of localised buckling of long struts [1,2], nonlinear optics [3], vibrating granular media [4], convection problems [5] and neuroscience [6,7]. In neural field models, stationary spatially localised regions of high activity ("bumps") have been studied in the context of working memory, as single-bump steady state solutions are believed to be the analogue of short-term memory [6,8-10]. Although these systems are quite diverse, they often display similar behaviour. Given this, it is of interest that the systems usually have key features in common, such as bistability, invariance under translation and spatial reflection, and are represented by differential equations that are at least fourth-order in space. The time-independent system can often be written as a dynamical

\footnotetext{
* Corresponding author. Tel.: +64 9373 7599; fax: +64 93737457.

E-mail address: a.elvin@math.auckland.ac.nz (A.J. Elvin).
}

system in space, where spatially localised solutions correspond to homoclinic orbits to the fixed point at the origin. Homoclinic snaking is also a feature in many systems [2,11-15], with some of the best studied examples being fourth-order partial differential equations $[1,2,15]$.

By exploiting the properties of higher order reversible Hamiltonian equations, advances have been made in the understanding of homoclinic solutions in pattern forming systems. One such example is the Swift-Hohenberg equation in both one and two spatial dimensions $[11-13,16]$. We refer the reader to the work of Champneys [1] for a review of both the theory and application of homoclinic orbits to equilibria in even-order reversible systems in four or more dimensions. Reversible and Hamiltonian systems have some important properties which we briefly discuss here. In a reversible, non-Hamiltonian system, symmetric homoclinic orbits are codimension zero. Therefore, they persist under a perturbation that preserves reversibility. However, asymmetric homoclinic orbits are codimension one and are destroyed by a generic perturbation that breaks the conserved quantity but still preserves reversibility [1]. In a non-reversible Hamiltonian system, both symmetric and asymmetric homoclinic orbits are codimensionzero. This is also true for a Hamiltonian-reversible system.

In this paper we extend the work of Laing and Troy [7,17] who found multiple bump solutions of a particular neural field model. We write the steady states of this model as solutions of a fourthorder reversible Hamiltonian ODE, and use these properties to investigate how steady states vary as parameters change. 
Laing and Troy $[7,17]$ studied the integro-differential equation

$\frac{\partial u(x, t)}{\partial t}=-u(x, t)+\int_{-\infty}^{\infty} w(x-y) f[u(y, t)] \mathrm{d} y$

where

$f(u)=2 \exp \left[-r /(u-\theta)^{2}\right] \Theta(u-\theta)$,

$\Theta$ is the Heaviside step function and

$w(x)=\mathrm{e}^{-b|x|}(b \sin |x|+\cos x)$.

Here, $u(x, t)$ is the average voltage, or activity level, of a neuronal population at spatial position $x$ and time $t$. The coupling function $w(x)$ is the distance-dependent strength of connectivity between neuronal elements and is even. The parameter $b$ governs the rate at which oscillations in $w$ decay with distance. The choice of $w$ was motivated by labelling studies showing that approximate periodic stripes are formed by coupled groups of neurons in the prefrontal cortex [18-20]. The firing rate function $f(u)$ models neurons firing once threshold is reached and tends to a maximal limit as the stimulus is increased. Parameter $\theta$ is the firing threshold and $r$ is the steepness parameter.

In [7], multiple bump steady states of (1), which are homoclinic orbits to the fixed point at the origin, were followed as $b$ was varied. By plotting the $\mathscr{L}^{2}$ norm of $u$ as a function of $b$, a "snaking" phenomenon was seen in the solution branches. "Snaking" has been seen previously in higher order scalar systems and in systems with homoclinic orbits [2,11-15]. The role of $r$ was briefly discussed, and it was found that increasing $r$ by 5\% led to a qualitative (although quite minor) change in the bifurcation diagram. This was further investigated by Elvin [21], who found large "breaks" in curves of solutions when $r$ was decreased by 5-10\%. In general, we expect a qualitative model such as (1) to be robust to small perturbations in parameters, therefore we want to understand what is causing the breaks. We show below that the steady states of (1)-(3) can be described by a four dimensional reversible Hamiltonian system. Our goal is to exploit the Hamiltonian structure and reversibility properties to explain the qualitative changes in the behaviour of the model, using two different approaches.

Firstly, we develop a numerical technique to find all homoclinic orbits of the system; these orbits correspond to the spatially localised steady states of (1)-(3). Numerically, we find a separate solution curve which exists when the firing rate function is sufficiently steep. This curve has not been reported previously and cannot be found using standard continuation techniques. Using level set analysis, we show that a codimension-one bifurcation, corresponding to the termination of solution curves, occurs at certain parameter values.

Secondly, we replace the firing rate function in (2) by a step function so that analytical techniques can be used to find travelling waves in the time-dependent system. We find the speed of travelling fronts, showing that stationary fronts exist at the same parameter values for which break-points in the solution curves exist. Using the Hamiltonian structure of the system we show that heteroclinic connections between fixed points also occur at the breakpoints.

The structure of the paper is as follows. In Section 2 we derive the ODE governing steady states of (1)-(3) and discuss its properties. In Section 3 we take a phase space approach and derive a map, certain solutions of which correspond to homoclinic orbits. The Hamiltonian structure is exploited in Section 4, while in Section 5 we discuss the consequences of replacing the firing rate function by the Heaviside step function. We conclude in Section 6 .

\section{The model and its properties}

The model in (1)-(3) supports spatially-uniform steady states, spatially-localised solutions such as homoclinic orbits and both stationary and travelling wave fronts (heteroclinic connections between a resting state and an excited state), and spatiallyperiodic patterns. We have previously shown the existence of both stable and transient spatially-periodic patterns beyond a Turing instability in (1)-(3) in one and two spatial dimensions [22] Both the non-trivial spatially uniform steady states, spatiallylocalised solutions and spatially-periodic solutions depend upon the parameter $b$.

For now, we consider time-independent solutions of (1) for which

$\lim _{|x| \rightarrow \infty}\left(u, u^{\prime}, u^{\prime \prime}, u^{\prime \prime \prime}\right)=(0,0,0,0)$,

i.e. stationary, spatially-localised solutions. These solutions satisfy the integral equation

$u(x)=\int_{-\infty}^{\infty} w(x-y) f[u(y)] \mathrm{d} y$.

Using the particular form of $w$ in (3), Eq. (5) can be transformed into a differential equation by the use of Fourier transforms.

\subsection{Derivation of $O D E$}

Noting that (5) involves a spatial convolution and taking the Fourier transform in space of this equation we obtain

$F[u]=F[w] F[f(u)]$

where $F[\cdot]$ denotes the Fourier transform. For $w$ as given by (3),

$F[w]=\frac{4 b\left(b^{2}+1\right)}{s^{4}+2 s^{2}\left(b^{2}-1\right)+\left(b^{2}+1\right)^{2}}$

where $s \in \mathbb{R}$ is the transform variable. Substituting (7) into (6) and rearranging we obtain

$\left(s^{4}+2 s^{2}\left(b^{2}-1\right)+\left(b^{2}+1\right)^{2}\right) F[u]=4 b\left(b^{2}+1\right) F[f(u)]$

which using Fourier transform identities can be written

$F\left[u^{\prime \prime \prime \prime}-2\left(b^{2}-1\right) u^{\prime \prime}+\left(b^{2}+1\right)^{2} u\right]=F\left[4 b\left(b^{2}+1\right) f(u)\right]$

where prime indicates derivative with respect to $x$. Applying inverse Fourier transforms to (8) we obtain the ODE

$u^{\prime \prime \prime \prime}-2\left(b^{2}-1\right) u^{\prime \prime}+\left(b^{2}+1\right)^{2} u=4 b\left(b^{2}+1\right) f(u)$

subject to the boundary conditions in (4). Solutions of (9) which are homoclinic to the origin correspond to spatially-localised steady states of (1). This technique of using Fourier transforms to convert integral equations to differential equations has been used several times before [6,17,23-26]. Eq. (9) has a number of important properties which we now discuss.

\subsection{Hamiltonian structure and reversibility}

Firstly, writing (9) as a system of four first-order ODEs and linearising them about the origin, we find that the Jacobian of this system has the four eigenvalues $\pm b \pm i$, i.e. the origin is a bifocus, with a two-dimensional unstable manifold and a two-dimensional stable manifold. Now (9) can be written as a reversible Hamiltonian system. For simplicity, write (9) as

$u^{\prime \prime \prime \prime}+a_{1} u^{\prime \prime}+a_{2} u+g(u)=0$ 
where $a_{1}=2\left(1-b^{2}\right), a_{2}=\left(b^{2}+1\right)^{2}$ and $g(u)=-4 b\left(b^{2}+1\right) f(u)$. Defining the variables $v, p_{u}$ and $p_{v}$ via

$u^{\prime}=v$

$v^{\prime}=p_{v}$

$p_{u}^{\prime}=a_{2} u+g(u)$

$p_{v}^{\prime}=-p_{u}-a_{1} v$

and defining the Hamiltonian

$H\left(u, v, p_{u}, p_{v}\right)=p_{u} v+\frac{p_{v}^{2}}{2}+\frac{a_{1} v^{2}}{2}-\frac{a_{2} u^{2}}{2}-G(u)$

where

$G(u) \equiv-8 b\left(b^{2}+1\right) \int_{0}^{u} \exp \left[-r /(z-\theta)^{2}\right] \Theta(z-\theta) \mathrm{d} z$,

we see that the Hamiltonian is conserved $\left(H^{\prime}=0\right)$ and the dynamics are given by

$u^{\prime}=\frac{\partial H}{\partial p_{u}}$

$v^{\prime}=\frac{\partial H}{\partial p_{v}}$

$p_{u}^{\prime}=-\frac{\partial H}{\partial u}$

$p_{v}^{\prime}=-\frac{\partial H}{\partial v}$.

Note that $G^{\prime}(u)=g(u)$. The system in (16)-(19) is invariant under the space-reversing symmetry

$R\left(x, u, v, p_{u}, p_{v}\right) \mapsto\left(-x, u,-v,-p_{u}, p_{v}\right)$,

that is, it is reversible.

\section{Using a map}

Having established the Hamiltonian structure of (9), we take a phase space approach and derive a two-dimensional map, certain solutions of which correspond to homoclinic orbits to the origin of (9). By doing this, we reduce the problem of finding single-bump homoclinic orbits to finding the zeros of a real scalar function.

\subsection{Derivation of a map}

We are interested in homoclinic orbits to the fixed point at the origin of (9). Homoclinic orbits of interest lie in $W^{s} \cap W^{u}$ where $W^{s}$ and $W^{u}$ denote the stable and unstable manifolds of the origin, respectively. In a Hamiltonian system, energy is conserved and the orbits of the system must lie on the energy surfaces. Therefore homoclinic orbits lie on the energy surfaces, or level sets, $H(u)=e \in \mathbb{R}$, where $e$ is a constant. Since $H=0$ at the origin, we only consider solutions on the zero energy surface, $H=0$, which reduces the dimension of the system under consideration from four to three. Since (9) is linear for $u<\theta$ we have explicit expressions for both the stable and unstable manifolds of the origin, and thus we need only consider the solutions of (9) for $\theta<u$. We choose two sections that effectively reduce the system dimension from three to two. Let $\Sigma_{0}$ be the two-dimensional section

$\Sigma_{0}=\left\{\left(u, v, p_{u}, p_{v}\right) \mid u=\theta, v>0, H=0\right\}$.

Now the value of $\left(v, p_{v}\right)$ defines a unique point on $\Sigma_{0}$, since if $H=0$ then $p_{u}$ can be found in terms of $u, v$ and $p_{v}$, using (15). We also define the section $\Sigma_{1}$ by

$\Sigma_{1}=\left\{\left(u, v, p_{u}, p_{v}\right) \mid u=\theta, v<0, H=0\right\}$.

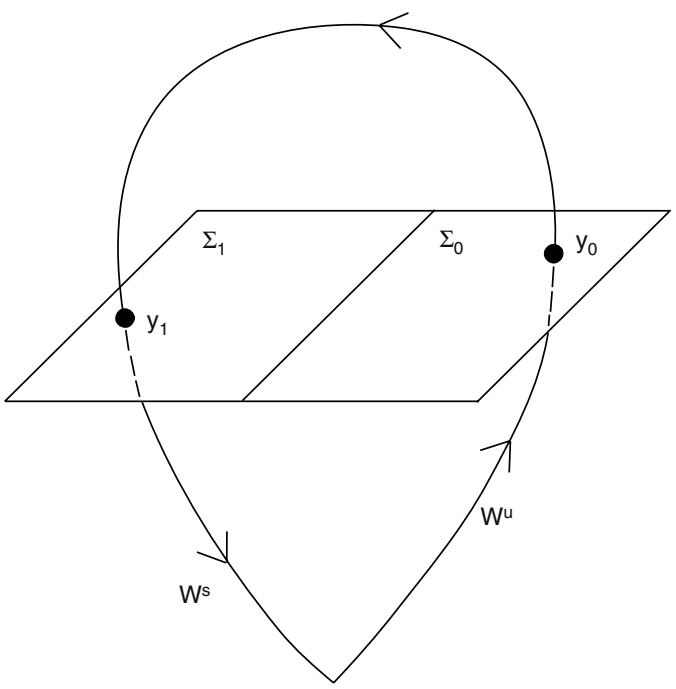

Fig. 1. Schematic of a homoclinic orbit. The orbit intersects the section $\Sigma_{0}$ transversally at the point $y_{0}$ and the section $\Sigma_{1}$ transversally at the point $y_{1}$. The stable and unstable manifolds of the fixed point are $W^{s}$ and $W^{u}$ respectively.

Note that $\Sigma_{0}$ and $\Sigma_{1}$ are two halves of a single plane (see Fig. 1). Now for $u<\theta$, solutions on the unstable manifold of the origin, $W^{u}$, can be written as

$u(x)=\mathrm{e}^{b x}(A \sin x+B \cos x)$

for some $A, B \in \mathbb{R}$. Using the translational invariance of the system we can choose $u(0)=\theta$, i.e. solutions on $W^{u} \cap \Sigma_{0}$ can be written

$u(x)=\mathrm{e}^{b x}(A \sin x+\theta \cos x)$

with $-b \theta<A$, since we need $0<v$ on $\Sigma_{0}$. Note that only one parameter, $A$, is needed to describe a point on $W^{u} \cap \Sigma_{0}$.

We define the mapping $P: \Sigma_{0} \rightarrow \Sigma_{1}$ for all $\mathbf{y}_{0} \in \Sigma_{0}$ as resulting from numerically integrating (9) with $\mathbf{y}_{\mathbf{0}}$ as an initial condition until the solution hits $\Sigma_{1}$ for the first time, at the point we define to be $\mathbf{y}_{1}$. In practice we will only consider points $\mathbf{y}_{\mathbf{0}} \in W^{u} \cap \Sigma_{0}$, where $W^{u} \cap \Sigma_{0}$ is a one-dimensional manifold. For such points, as long as $-b \theta<A$, the solution of (9) through $\mathbf{y}_{\mathbf{0}}$ will always transversally meet $\Sigma_{1}$ for some $x>0$, and thus $P$ is defined for these $\mathbf{y}_{\mathbf{0}}$. From (20) our initial condition for (9) is

$$
\left(\begin{array}{c}
u(0) \\
u^{\prime}(0) \\
u^{\prime \prime}(0) \\
u^{\prime \prime \prime}(0)
\end{array}\right)=\left(\begin{array}{c}
\theta \\
A+b \theta \\
-\theta+2 A b+b^{2} \theta \\
-A-3 b \theta+3 b^{2} A+b^{3} \theta
\end{array}\right)
$$

which can be written in Hamiltonian coordinates using (11)-(14) as

$\left(\begin{array}{c}u(0) \\ v(0) \\ p_{u}(0) \\ p_{v}(0)\end{array}\right)=\left(\begin{array}{c}u(0) \\ u^{\prime}(0) \\ -a_{1} u^{\prime}(0)-u^{\prime \prime \prime}(0) \\ u^{\prime \prime}(0)\end{array}\right)$.

Using (21)-(22) we can write

$W^{u} \cap \Sigma_{0}=\left\{\left(u, v, p_{u}, p_{v}\right) \mid u=\theta, v>0, H=0\right.$,

$$
\left.p_{v}=2 b v-\theta\left(b^{2}+1\right)\right\}
$$

which can be visualised as a straight line in the right half of the $\left(v, p_{v}\right)$ plane. Because of the reversibility of the system, the stable manifold of the origin, $W^{s}$, is given by $W^{s}=R\left(W^{u}\right)$. Thus

$W^{s} \cap \Sigma_{1}=\left\{\left(u, v, p_{u}, p_{v}\right) \mid u=\theta, v<0, H=0\right.$,

$$
\left.p_{v}=-2 b v-\theta\left(b^{2}+1\right)\right\}
$$




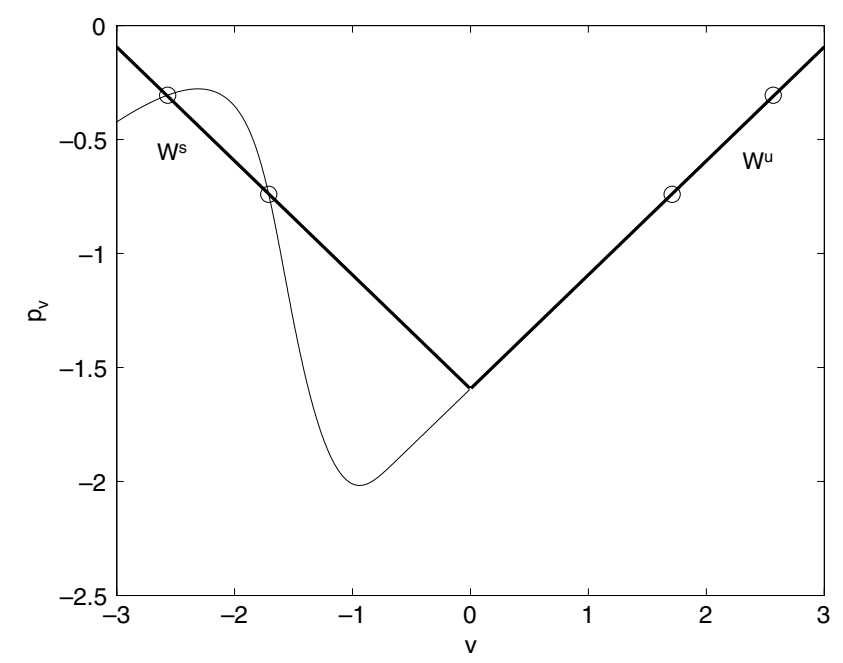

Fig. 2. Mapping of initial conditions on $W^{u} \cap \Sigma_{0}$ for parameter values $(b, r, \theta)=$ $(0.25,0.095,1.5)$. The circles on $W^{u} \cap \Sigma_{0}$ indicate the two initial conditions that map to the two circles on $W^{s} \cap \Sigma_{1}$. The two initial conditions lie on two symmetric homoclinic orbits.

which forms another straight line in the left half of the $\left(v, p_{v}\right)$ plane.

To find homoclinic orbits to the origin with $u>\theta$ over only one interval (a 1-bump solution), we choose a $\mathbf{y}_{\mathbf{0}} \in W^{u} \cap \Sigma_{0}$ and let $\mathbf{y}_{1}=P\left(\mathbf{y}_{0}\right)$. If $\mathbf{y}_{1} \in W^{S} \cap \Sigma_{1}$ then $\mathbf{y}_{\mathbf{0}}$ lies on such a homoclinic orbit, as shown in Fig. 1. Let the coordinates of $\mathbf{y}_{\mathbf{0}} \in W^{u} \cap \Sigma_{0}$ be $\left(v^{0}, p_{v}^{0}\right)$ and $\mathbf{y}_{1} \in W^{s} \cap \Sigma_{1}$ be $\left(v^{1}, p_{v}^{1}\right)$, and suppose that these points lie on a homoclinic orbit to the origin. If $v^{0}=-v^{1}$ then $\mathbf{y}_{\mathbf{0}}$ lies on a symmetric homoclinic orbit, otherwise it is on an asymmetric orbit. In practice for a general $\mathbf{y}_{\mathbf{0}} \in W^{u} \cap \Sigma_{0}$ we calculate the signed vertical distance, $h$, between $P\left(\mathbf{y}_{\mathbf{0}}\right)$ and $W^{S} \cap \Sigma_{1}$ :

$h=p_{v}^{1}-\left[-2 b v^{1}-\theta\left(b^{2}+1\right)\right]=p_{v}^{1}+2 b v^{1}+\theta\left(b^{2}+1\right)$.

Recalling that a point on $W_{u} \cap \Sigma_{0}$ can be parameterised by $A$ we can regard $h$ as being a scalar function of $A$; to find homoclinic orbits to the origin we just need to find zeros of $h(A)$. Note that any $N$-bump solution can be found by modifying the mapping $P$ to terminate on the $N$ th intersection of $\Sigma_{1}$, crossing in the appropriate direction.

\subsection{Numerical results}

For all of the analyses in this section we set $\theta=1.5$. We initially set $r=0.095$. By varying $b$, we use the mapping derived above to search different regions of parameter space and find all existing homoclinic orbits. For $b=0.25$, we find that the mapping is continuous, as seen in Fig. 2 where a plot of $W^{s} \cap \Sigma_{1}, W^{u} \cap \Sigma_{0}$ and the mapping of initial conditions on $W^{u} \cap \Sigma_{0}$ are shown. The circles on $W^{u} \cap \Sigma_{0}$ indicate the two initial conditions which map to $W^{s} \cap$ $\Sigma_{1}$ (also indicated by circles), therefore these two initial conditions lie on two homoclinic orbits. The orbits are symmetric as the $p_{v}$ coordinate of each initial condition on $W^{u} \cap \Sigma_{0}$ is unchanged under the mapping to $W^{s} \cap \Sigma_{1}$.

To find the solution curves, we convert the solutions found with the mapping to full solutions over the finite domain $x \in$ $[-15 \pi, 15 \pi]$, as with this size domain the boundary conditions in (4) are satisfied. The software package AUTO [27] is used for continuation and solving bifurcation problems in ordinary differential equations with one or more free parameters and includes the package HomCont for the bifurcation analysis of homoclinic orbits. Therefore we write the ODE in (9) as a system of first order equations, take a full solution as a starting solution, and use AUTO to compute the solution curves by varying the parameter $b$.
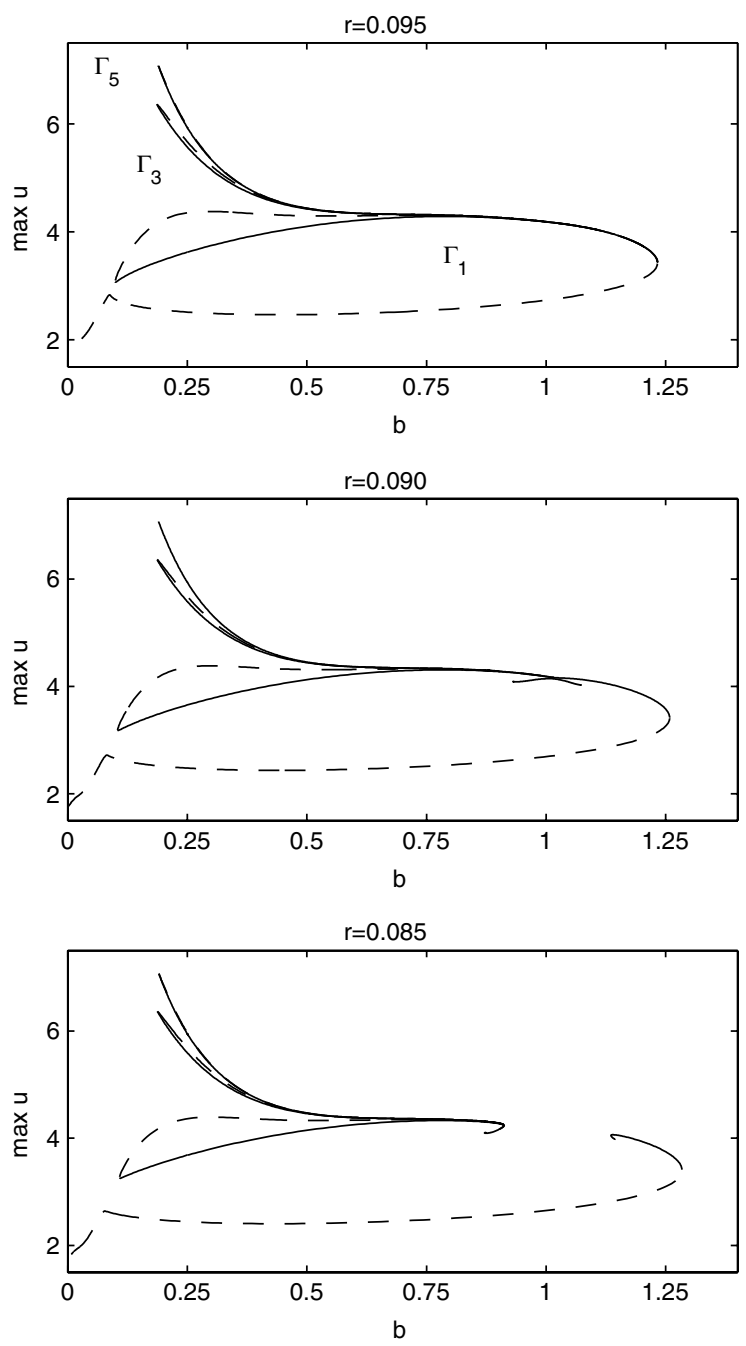

Fig. 3. Solution curves of homoclinic orbits for (1)-(3) with $b$ the continuation parameter. Top: For $r=0.095$, the main solution curve is continuous. Middle: For $r=0.090$, a small separate solution curve exists and lies near, but does not quite touch, the solution curve of stable homoclinic orbits. Bottom: For $r=0.085$, the small solution curve has met the main curve, causing a large "break" where no stable homoclinic orbits exist. Solid line: stable, dashed: unstable. See the text for an explanation of labels.

We find the solution curves shown in the top plot of Fig. 3 where the global maximum of $u$ is plotted as a function of $b$. The symbols $\Gamma_{1}, \Gamma_{3}$ and $\Gamma_{5}$ indicate families of 1 -, 3- and 5-bump solutions respectively. The results agree with the solution curves found in [7] where there are two single-bump symmetric homoclinic orbits at $b=0.25$. We vary $b$ but no new solution curves are found using our two-dimensional mapping (for $r=0.095$ ) and the solution curves are continuous. Solid (dotted) lines show branches of stable (unstable) solutions. Solution stability is determined numerically using an eigenvalue analysis of the spatially perturbed full system [21]. We see that the system is multi-stable as $N$-bump solutions, in general, come in pairs of one stable solution and one unstable solution.

We now decrease $r$ to 0.090 . By varying $b$ and using our mapping, we find unexpected solutions which lie on a separate curve inside the main solution curve, centred approximately about $b=1$ (middle plot of Fig. 3). The small separate curve lies very near, but does not quite touch, the main solution curve, which is still continuous. This separate solution curve has not been reported previously in the literature and could not be found using standard continuation methods, as we previously had no known solution on the small curve. 

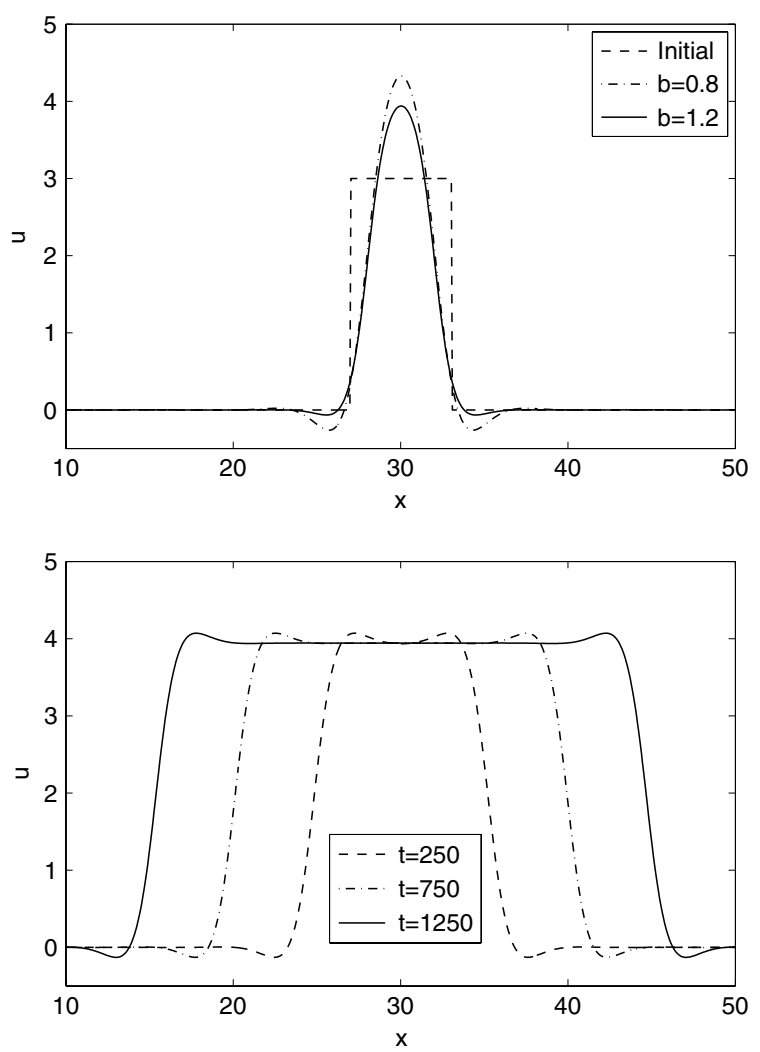

Fig. 4. Top: initial condition (dashed) and steady states of a simulation of (1)-(3) for $b=0.8$ (dash-dotted) and $b=1.2$ (solid). Bottom: snapshots at the specified times when $b=1$. Other parameters: $r=0.085, \theta=1.5$.

As $r$ decreases (making $f$ steeper), the separate solution curve meets the main solution curve at $(b, r)=(1.0167,0.0899)$ ( 4 d.p.). As we decrease $r$ further, a "break" develops in the main solution curve and the gap widens with a spiral terminating each side of the break. In the bottom plot of Fig. $3(r=0.085)$, there is a very clear gap where no stable homoclinic orbits exist. The terminating ends of the break in the solution curve appear to end in spirals. It has not been possible to numerically determine the stability of the solutions on spirals, however, we expect that stability changes in saddle-node bifurcations.

Fig. 4 shows results from simulations of the full system (1)-(3) as $b$ is varied across the break seen in the bottom panel of Fig. 3 . We use the same initial condition (shown in the top panel of Fig. 4) and three different values of $b$. For $b=0.8$ and 1.2 we find stable 1-bump solutions but for $b=1$ (i.e. in the break seen in the lower panel of Fig. 3), two fronts travelling in opposite directions are observed, resulting in an expanding region of high activity.

In Fig. 5 we take a closer look at the small curve that exists for $r=0.090$ and see that a kink has appeared in the main solution curve near $b=1$ where the distance between the main curve and the small curve is at a minimum. At $r=0.095$, the small solution curve did not exist and there was no kink in the main solution curve. The ends of the small solution curve seem to terminate in spirals. Fig. 6 shows the intersection of the small solution curve with the main solution curve at $(b, r)=(1.0167,0.0899)$ (4 d.p.) where two tails ending in spirals are formed. These two tails become the terminating ends on each side of the break in the main solution curve as $r$ decreases from 0.0899 . The main solution curve breaks around $b=1$ and here, the coefficient of the second derivative in the ODE in (9) vanishes. We plot solutions at $b=$ 0.932 for $r=0.090$ and $r=0.0899$ in Fig. 7 to see if there are qualitative differences in the solutions. In the left plot we show solutions from the main curve (solid line) and the small curve

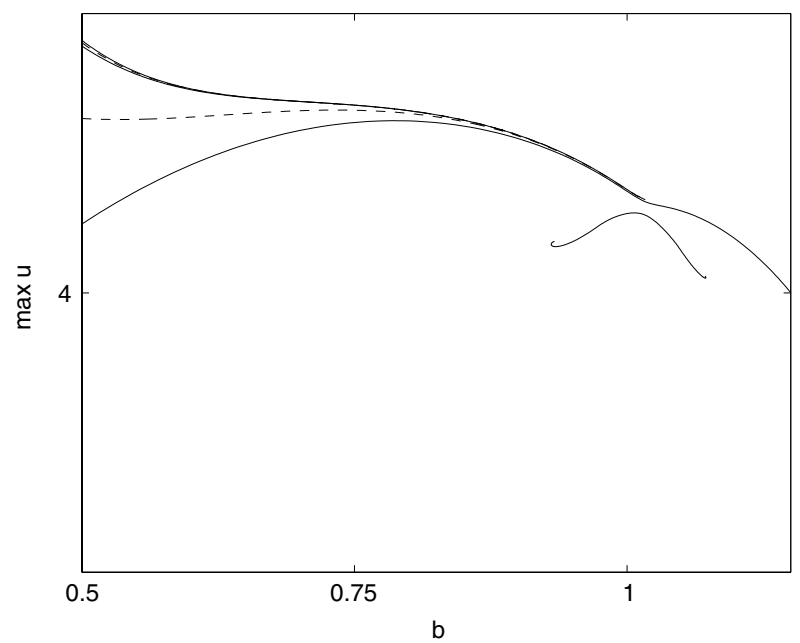

Fig. 5. Closer view of the small separate solution curve in the middle plot of Fig. 3 for $r=0.090$. There is a kink in the main solution curve where the distance to the small curve is smallest.

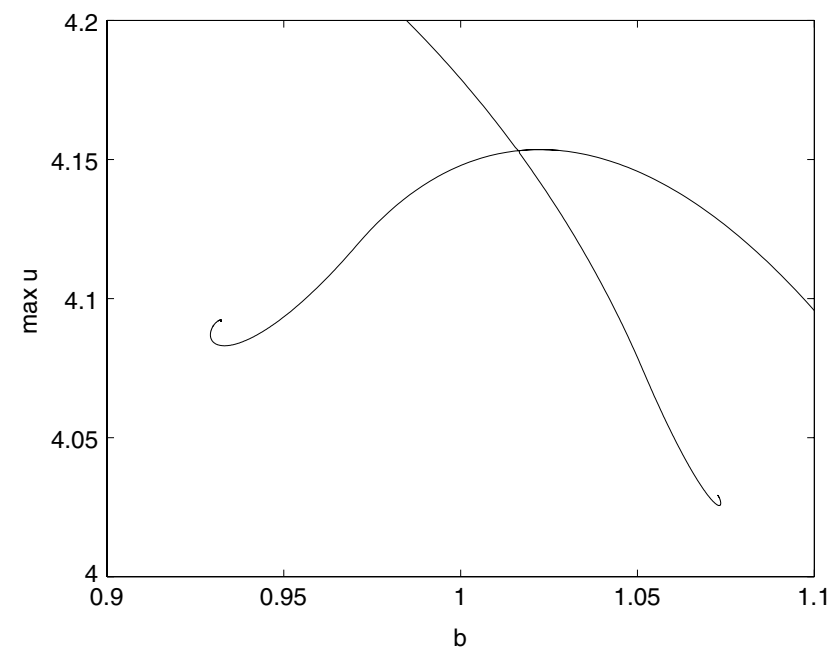

Fig. 6. As $r$ decreases from 0.090 to 0.0899 , the small separate solution curve shown in Fig. 5 meets the main solution curve at $b=1.0167$. A closer view of the intersection between the two solution curves shows that two tails ending in spirals have formed.

(dotted line) from Fig. 5 for $r=0.090$. At this particular value of $b$, the solution on the small curve is near the terminating spiral and is a "dimple" bump solution, that is, the solutions have a positive second spatial derivative at the centre of the bump. The solution is also broader than the single-bump solution on the main curve. In the right plot of Fig. 7 we do the same for the main curve (solid line) and the left tail formed by the small curve (dotted line) in Fig. 6 for $r=0.0899$. Again, near the terminating spiral on the tail, the solution is a "dimple" bump and is a broader solution than that on the main curve.

We are interested in how the small branch of solutions from Fig. 5 changes as $r$ is varied. In Fig. 8 we plot the curve for five different values of $r$. The topmost curve is the small solution curve at $r=0.090$. Continuation methods reveal a shrinking of this curve as $r$ increases beyond 0.090. At $r=0.091875$, the two endpoints of the curve meet, creating an isola [28]. The isola exists only briefly and disappears quickly as $r$ increases further. Therefore the small solution curve only exists for a small range of $r$. To see how the solutions change on the curves in Fig. 8, we plot the solution at $b=1$ from four of the curves in Fig. 9. As the small curve shrinks, the solution becomes progressively broader and eventually, on the 

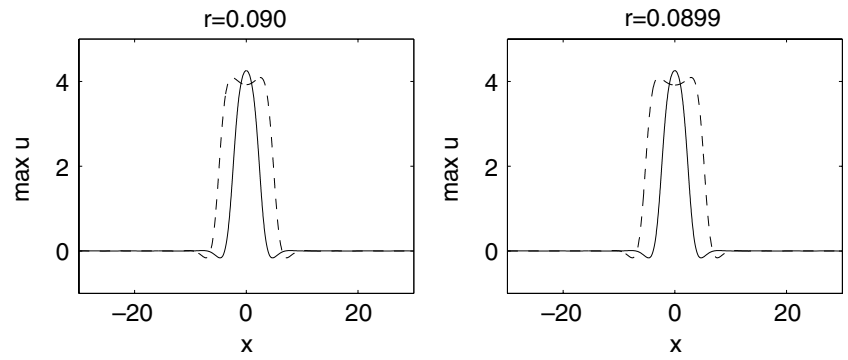

Fig. 7. Left: Solutions at $b=0.932$ for $r=0.090$ on the main solution curve of single-bump solutions (solid line) and the small solution curve (dotted line) in Fig. 5. Right: Solutions at $b=0.932$ for $r=0.0899$ on the upper curve of single-bump solutions (solid line) and the small tail caused by the small curve meeting the main curve (dotted line) in Fig. 6 . In both cases, the solutions on the small curve near the terminating spiral are "dimple" bumps that become broader the closer they are to the spiral.

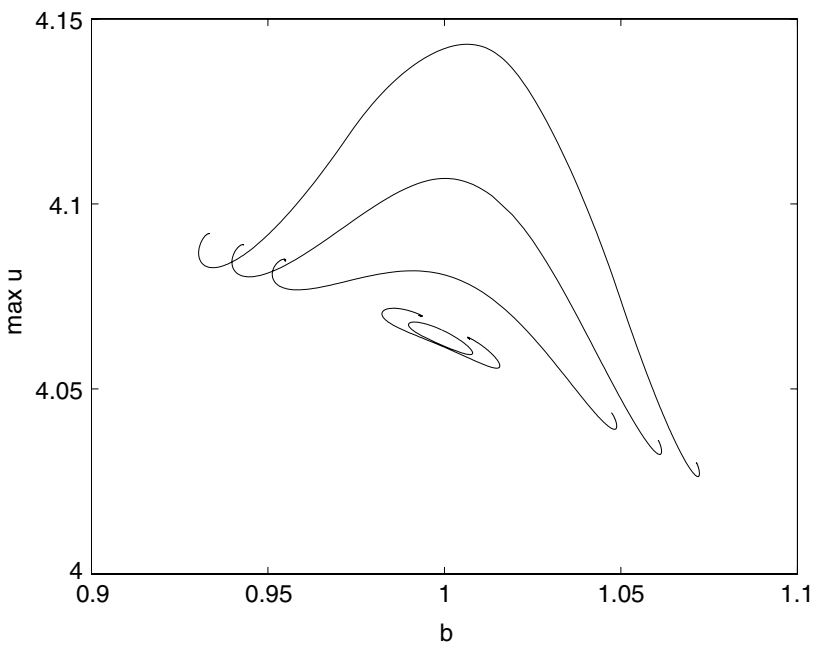

Fig. 8. The topmost curve is the small curve found for $r=0.090$ in Fig. 5. As $r$ increases to $0.0905,0.0910,0.0918$ and 0.091875 , the curve shrinks and eventually forms an isola at $r=0.091875$.

isola that exists at $r=0.091875$, becomes a "dimple" bump. All solutions on the isola are "dimple" bump solutions.

We now know how this particular branch of solutions is created, that it eventually meets the main curve, causing it to break as $r$ decreases and creating a gap in the main solution curve where no stable homoclinic orbits exist.

Although the mapping reduces the problem of finding homoclinic orbits to finding the zeros of a real scalar function, the function obtained is not necessarily continuous, so it can be difficult to make conclusions about the global existence of solutions and global bifurcations. The existence of a small separate branch of solutions partially explains the gap in the curve, but we want to understand why the curves end and what causes the gap to widen as $f$ becomes steeper. In the next section, we use level set analysis to find a global bifurcation at the terminating ends of the solution curves.

\section{Heteroclinic connections}

Homoclinic orbits lie on energy surfaces, and therefore the topology of the level sets $\{u: H(u)=e, e \in \mathbb{R}\}$ can change only where the level set contains a critical point. These critical points are the fixed points of the system. Up until now, we have only considered homoclinic orbits to the fixed point at the origin; we now study other fixed points of the system to understand what causes the curve of solutions to break and destroy homoclinic orbits to the origin.
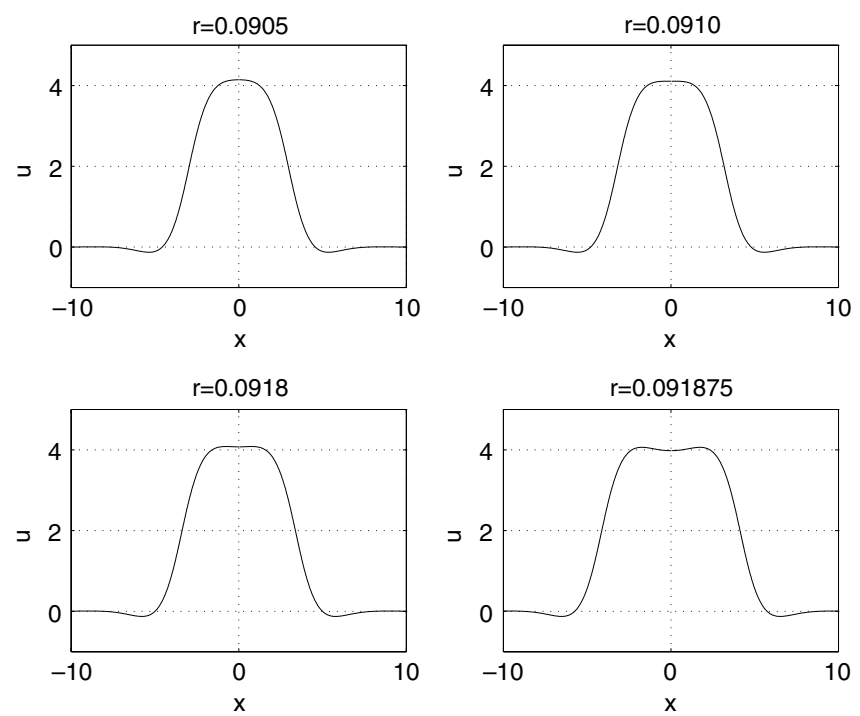

Fig. 9. Solutions at $b=1$ from the curves for $r=0.0905,0.0910,0.0918$ and 0.091875 in Fig. 6 . As the small curve shrinks ( $r$ increases), the solutions become progressively broader and on the isola at $r=0.091875$, the solutions have become "dimple" bumps.

\subsection{Theory}

Depending on parameter values, (9) can have up to three fixed points. One is the origin, $\mathbf{Z}_{\mathbf{0}}$, which exists when $0<\theta$. The other two are referred to as $\mathbf{Z}_{\mathbf{1}}$ for which $\left(u, u^{\prime}, u^{\prime \prime}, u^{\prime \prime \prime}\right)=\left(u_{1}, 0,0,0\right)$ and $\mathbf{Z}_{2}$, for which $\left(u, u^{\prime}, u^{\prime \prime}, u^{\prime \prime \prime}\right)=\left(u_{2}, 0,0,0\right)$, where $u_{1}<u_{2}$ and $u_{1}$ and $u_{2}$ are both solutions of

$u=\frac{8 b \exp \left[-r /(u-\theta)^{2}\right]}{b^{2}+1}$.

Homoclinic orbits to the origin lie on the zero energy surface, the level set $H=0$, and can only be destroyed if this level set contains a fixed point. Using (15), we find $H$ at $\mathbf{Z}_{\mathbf{1}}$ and $\mathbf{Z}_{\mathbf{2}}$ as a function of $b$ for the three different values of $r: r=0.095,0.090,0.085$, as in Section 3.2. By doing this we can determine if either of the nonzero fixed points can meet the zero energy surface, $H=0$. Note that the value of $H$ at $\mathbf{Z}_{\mathbf{1}}$ and $\mathbf{Z}_{\mathbf{2}}$ must be found numerically.

\subsection{Results}

Fig. 10 shows the value of the Hamiltonian $(H)$ at the two nontrivial fixed points as a function of $b$ (bottom row) and solution curves (top row) as a function of $b$ for three different values of $r$. For $r=0.095$ (left column), both $H\left(\mathbf{Z}_{\mathbf{1}}\right)$ and $H\left(\mathbf{Z}_{\mathbf{2}}\right)$ are negative for all $b$ and the solution curves are continuous. For $r=0.090$ (middle column), there are two values of $b$ for which $H\left(\mathbf{Z}_{\mathbf{2}}\right)=0$ and these values of $b$ correspond to the endpoints of the small separate solution curve in the top panel. So there are two values of $b$ for which the fixed point $\mathbf{Z}_{2}$ meets the zero energy surface $H=0$. For $r=0.085$ (right column), there is a large break in the main solution curve and the terminating ends of the break correspond to the values of $b$ for which $H\left(\mathbf{Z}_{2}\right)=0$.

For parameter values such that $H\left(\mathbf{Z}_{\mathbf{2}}\right)=0$, a codimension-one bifurcation occurs in which the stable and unstable manifolds of $\mathbf{Z}_{\mathbf{0}}$ and $\mathbf{Z}_{\mathbf{2}}$ intersect, destroying all homoclinic orbits to the origin. This bifurcation is similar to the codimension-two heteroclinic bifurcation called a $T$-point or terminal point, studied in twodimensional parameter space in the Lorenz equations [29]. In our system, as we move closer to the break points in the solution curves shown in Fig. 10, the solutions spend longer near the fixed point $\mathbf{Z}_{2}$ and thus develop a broad "plateau" in their centre. At each break 

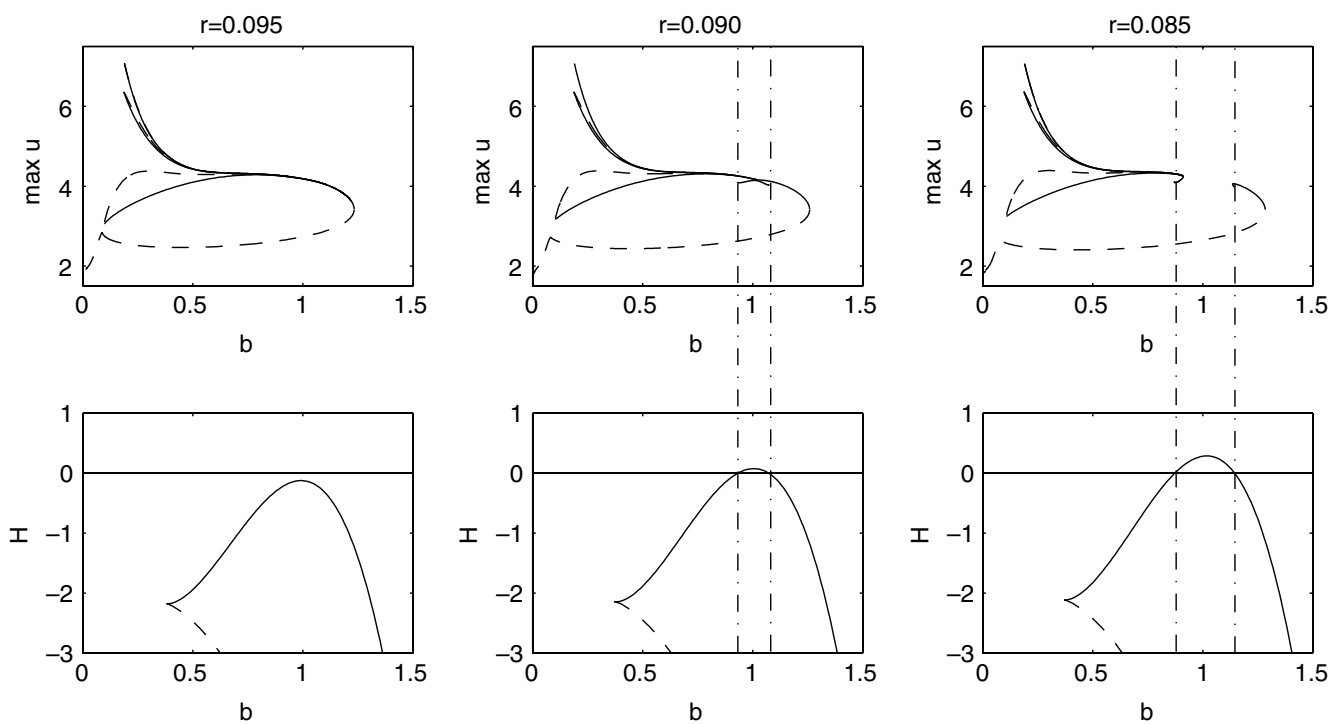

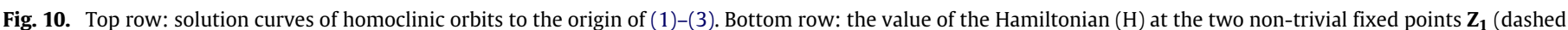
line) and $\mathbf{Z}_{\mathbf{2}}$ (solid line). Columns from left to right: $r=0.095, r=0.090, r=0.085$. Dash-dot vertical lines indicate values of $b$ for which $H\left(\mathbf{Z}_{2}\right)=0$.

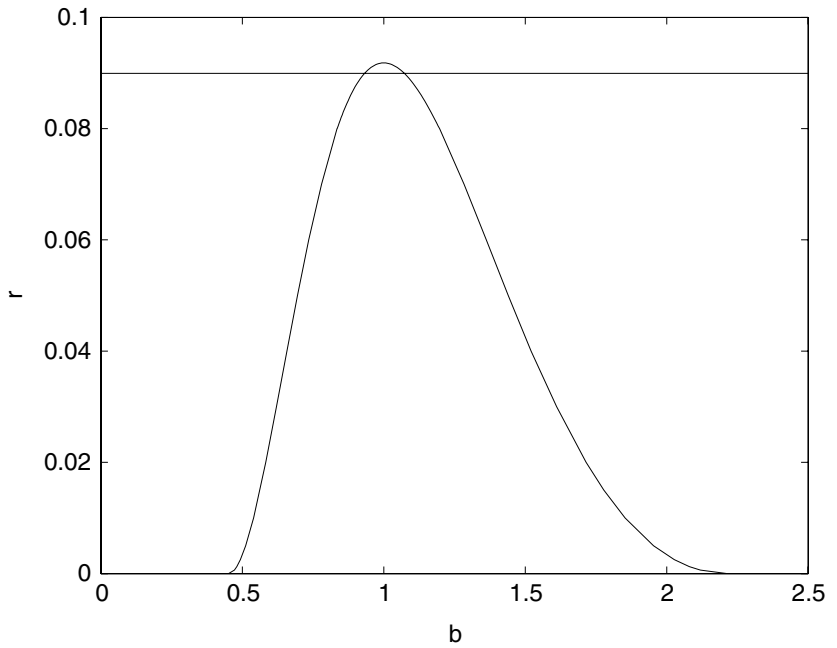

Fig. 11. Points in $(b, r)$ parameter space where $H\left(\mathbf{Z}_{\mathbf{2}}\right)=H\left(\mathbf{Z}_{\mathbf{0}}\right)=0$ for $\theta=1.5$ in (1)-(3). At $r=0.0899$ (horizontal line), the separate solution curve meets the main solution curve. Below this line, the curve gives the endpoints of the solution curve. Above this line, the curve gives the terminating ends of the separate solution curve.

point, $\mathbf{Z}_{\mathbf{2}}$ intersects the homoclinic orbit, forming a heteroclinic orbit between $\mathbf{Z}_{\mathbf{2}}$ and the origin.

We conjecture that the end points of the solution curves occur where $H\left(\mathbf{Z}_{\mathbf{2}}\right)=H\left(\mathbf{Z}_{\mathbf{0}}\right)=0$ as this is the behaviour we have seen for $r=0.085$ and $r=0.090$. Fig. 11 shows the curve in $(b, r)$ parameter space where $H\left(\mathbf{Z}_{\mathbf{2}}\right)=H\left(\mathbf{Z}_{\mathbf{0}}\right)=0$. At $r=0.0899$ (4 d.p.), the separate solution curve meets the main curve. In Fig. 11 the horizontal line at $r=0.0899$ intersects the plotted curve at the two values of $b$ where the endpoints of the small curve occur. Above the horizontal line, the small separate solution curve exists for a small range of $r>0.0899$ and the plotted curve gives the endpoints of this separate solution curve. Below the horizontal line, the separate solution curve no longer exists and the plotted curve gives the the terminating ends of the main solution curve.

\section{Heaviside firing rate function}

So far, many of our results have had to be calculated numerically, due to the presence of the nonlinear function (2). More ana- lytical progress can be made if the firing rate function $f$ is replaced by a piecewise linear function [6] or a Heaviside step function $[8,24]$. Here we consider the case when $f(u)=2 \Theta(u-\theta)$, which is the result of taking $r \rightarrow 0$ in (2).

In most neural field models, some form of lateral inhibition is required for stable stationary bumps to exist $[8,10]$. It has been shown previously that spike-frequency adaptation changes travelling waves from fronts to bumps in a one-dimensional single population model [30]. More recently, Kilpatrick and Bressloff [31] found that stable stationary bumps can coexist with fronts in an excitatory neuronal network with synaptic depression, however, bumps cannot exist in the presence of adaptation. In this section we show that our model, with decaying oscillatory connectivity but no negative feedback, can support travelling waves in the form of fronts as well as stable stationary bumps.

\subsection{Using a map}

The derivation of a map can be carried out in a similar way to that in Section 3.1, the only difference being that we can now analytically find $u(x)$ when $\theta<u(x)$, rather than having to numerically integrate (9). The solutions found are shown in Fig. 12, and there is a wide range of $b$ for which no stable homoclinic orbits can be found. The values of $b$ for which the solution curves terminate agree with the values of $b$ corresponding to $r=0$ in Fig. 11. Thus it seems that the terminating ends of the solution curves for $r=0$ must occur when a nonzero fixed point of the system meets the zero energy surface. We now investigate this further by finding heteroclinic orbits of the system.

\subsection{Heteroclinic connections}

As before, we find fixed points of (9). The origin is a fixed point for $\theta>0$ and there exists one other, $\hat{\mathbf{z}}$, for which $\left(u, u^{\prime}, u^{\prime \prime}, u^{\prime \prime \prime}\right)=$ $\left(8 b /\left(b^{2}+1\right), 0,0,0\right)$, when $0<\theta<8 b /\left(b^{2}+1\right)$. Using (15) we see that at $\hat{\mathbf{Z}}, H=32 b^{2}-8 b\left(b^{2}+1\right) \theta$, thus $\hat{\mathbf{Z}}$ will lie on the level set $H=0$ when

$\theta=\frac{4 b}{b^{2}+1}$.

Setting $\theta=1.5$ in (23) we find that this equation is satisfied when $b=0.4514$ or 2.2153 ( 4 d.p.) which are exactly the endpoints of 


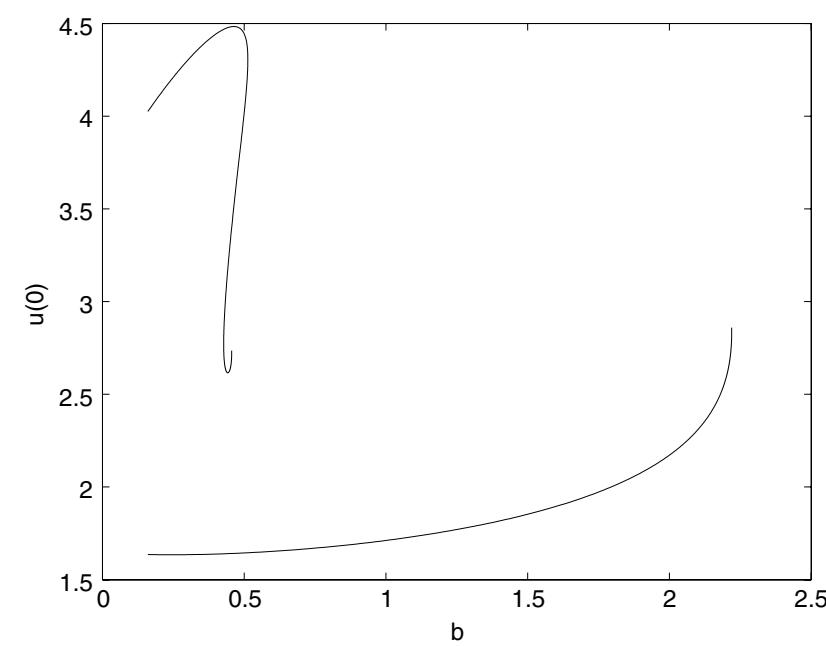

Fig. 12. Single-bump solution curves of (1) and (3) with $f(u)=2 \Theta(u-1.5)$.

the curves in Fig. 12 and also the values of $b$ at which the curve in Fig. 11 touches the $b$ axis. Furthermore, Eq. (23) cannot be satisfied by any real $b$ if $\theta>2$, which implies that the curve in Fig. 12 will not break if $\theta>2$. It appears that the breaks in the solution curve are related to the firing threshold, $\theta$.

\subsection{Stationary fronts}

The analysis so far has been concerned with time-independent solutions of (1)-(3), which satisfy the ODE (9). However, it is also possible that (1)-(3) supports travelling fronts or pulses. A wave travelling with constant speed is stationary in a coordinate frame moving with an appropriate constant speed, so we now study timedependent solutions of (1), utilizing a moving coordinate frame.

\subsubsection{Front construction}

Following [32], by using a Green's function in time the solution of (1) can be written in integral form

$u(x, t)=\int_{-\infty}^{\infty} \int_{0}^{\infty} \eta(s) w(y) f[u(x-y, t-s)] \mathrm{d} s \mathrm{~d} y$

where $\eta(t)=\Theta(t) \mathrm{e}^{-t}$, and this form can be used to construct travelling wave solutions. We now set $f(u)=2 \Theta(u-\theta)$ and explicitly construct travelling front solutions that join the states $u=0$ and $u=8 b /\left(b^{2}+1\right)$. For certain parameter values these fronts are stationary, and these parameter values are found to be those for which heteroclinic connections between the origin and $\hat{\mathbf{Z}}$ were found in Section 5.2.

Define the travelling coordinate $\xi=x-c t$, where $c$ is a speed, and let $U(\xi, t)=u(x-c t, t)$. Then (24) can be written

$U(\xi, t)=\int_{-\infty}^{\infty} \int_{0}^{\infty} \eta(s) w(y) f[U(\xi-y+c s, t-s)] \mathrm{d} s \mathrm{~d} y$.

A travelling wave solution of (24) is a time-independent solution of $(25)$, say $q(\xi)$, which satisfies

$q(\xi)=\int_{-\infty}^{\infty} \int_{0}^{\infty} \eta(s) w(y) f[q(\xi-y+c s)] \mathrm{d} s \mathrm{~d} y$.

If we define

$\phi(\xi) \equiv \int_{-\infty}^{\infty} w(y) f[q(\xi-y)] \mathrm{d} y$

then (26) can be written

$q(\xi)=\int_{0}^{\infty} \eta(s) \phi(\xi+c s) \mathrm{d} s$.

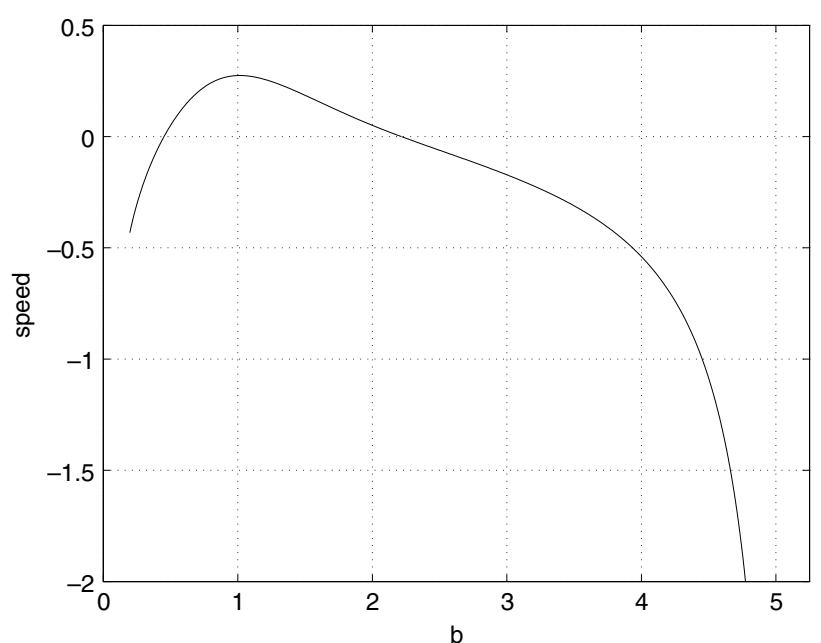

Fig. 13. Speed of travelling fronts, $c$, in (28) as a function of $b$ for $\theta=1.5$. Stationary fronts occur at $b=0.451$ and $b=2.215$. (Note that the curve does not exist for $b<0.195$ because for a front of the type constructed to occur we must have $\left.\theta<8 b /\left(b^{2}+1\right)\right)$.

Suppose that $\theta<q(\xi)$ for $\xi<0, q(0)=\theta$ and $q(\xi)<\theta$ for $0<\xi$, i.e. $q$ is a front. Then

$\phi(\xi)=2 \int_{\xi}^{\infty} w(y) \mathrm{d} y$.

Since $q(0)=\theta$, we see from (28) that

$\theta=\int_{0}^{\infty} \eta(s) \phi(c s) \mathrm{d} s$

which can be solved for the speed $c$ in terms of other parameters [21]. Stationary fronts satisfy

$\theta=\int_{0}^{\infty} \eta(s) \phi(0) \mathrm{d} s=2 \int_{0}^{\infty} w(y) \mathrm{d} y=\frac{4 b}{b^{2}+1}$

since $\int_{0}^{\infty} \eta(s) d s=1$, which is the same expression as found in (23). Using the expression for travelling fronts in (28), we find the front speed, $c$, as a function of $b$. The results are shown in Fig. 13. Stationary fronts exist at $b=0.451$ and $b=2.215$ which are the same values of $b$ at which the endpoints of the solution curves in Fig. 12 occur.

\subsubsection{Front stability}

The stability of the travelling fronts just constructed can be determined by linearising the dynamics about them and constructing an Evans function, as has been done previously for neural field models with a Heaviside firing rate function [24,30,32-34]. We first let $U(\xi, t)=q(\xi)+u(\xi, t)$ where $q(\xi)$ satisfies (26), and expand (25) to first order in $u$, obtaining

$$
\begin{aligned}
u(\xi, t)= & \int_{-\infty}^{\infty} \int_{0}^{\infty} \eta(s) w(y) f^{\prime}[q(\xi-y+c s)] \\
& \times u(\xi-y+c s, t-s) \mathrm{d} s \mathrm{~d} y .
\end{aligned}
$$

To find bounded continuous solutions on $\mathbb{R}$ for each $t$ we look for solutions of the form $u(\xi, t)=u(\xi) \mathrm{e}^{\lambda t}$. Substituting this into (32) we obtain

$$
\begin{aligned}
u(\xi)= & \frac{1}{c} \int_{-\infty}^{\infty} \int_{\xi-y}^{\infty} \eta(s / c-\xi / c+y / c) w(y) f^{\prime}[q(s)] \\
& \times u(s) \mathrm{e}^{-\lambda(s-\xi+y) / c} \mathrm{~d} s \mathrm{~d} y .
\end{aligned}
$$


Defining $z=q(s)$ and recalling that $f^{\prime}(z)=2 \delta(z-\theta)$, (33) can be written

$$
\begin{aligned}
u(\xi)= & \int_{-\infty}^{\infty} \int_{q(\xi-y)}^{q(\infty)} \eta\left(q^{-1}(z) / c-\xi / c+y / c\right) w(y) \mathrm{e}^{-\lambda\left(q^{-1}(z)-\xi+y\right) / c} \\
& \times \frac{2 \delta(z-\theta)}{c\left|q^{\prime}\left(q^{-1}(z)\right)\right|} u\left(q^{-1}(z)\right) \mathrm{d} z \mathrm{~d} y,
\end{aligned}
$$

and using the fact that $q^{-1}(\theta)=0$, this simplifies to

$u(\xi)=\frac{2 u(0)}{c\left|q^{\prime}(0)\right|} \int_{-\infty}^{\infty} \eta(y / c-\xi / c) w(y) \mathrm{e}^{-\lambda(y-\xi) / c} \mathrm{~d} y$.

Evaluating (35) at $\xi=0$, using the fact that $\eta(y)=0$ for $y<0$, and rescaling $y$ we obtain

$u(0)=\frac{2 u(0)}{\left|q^{\prime}(0)\right|} \int_{0}^{\infty} \eta(y) w(c y) \mathrm{e}^{-\lambda y} \mathrm{~d} y$,

which has nontrivial solutions only if $\mathcal{E}(\lambda)=0$, where

$\mathcal{E}(\lambda)=1-\frac{2}{\left|q^{\prime}(0)\right|} \int_{0}^{\infty} \eta(y) w(c y) \mathrm{e}^{-\lambda y} \mathrm{~d} y$.

We identify $\mathscr{E}(\lambda)$ as the Evans function, and its roots are the isolated eigenvalues associated with the linearisation of (25) about the travelling wave solution $q$. It is straightforward to show that $\mathcal{E}(0)=0$, as expected, reflecting the translational invariance of the problem. Defining

$\mathcal{H}(\lambda)=\int_{0}^{\infty} \eta(y) w(c y) \mathrm{e}^{-\lambda y} \mathrm{~d} y$

and using the fact that $\varepsilon(0)=0$, one can write

$\mathscr{E}(\lambda)=1-\frac{\mathscr{H}(\lambda)}{\mathscr{H}(0)}$

thus avoiding the explicit construction of $q^{\prime}(0)$. When $c=0$, $\varepsilon(\lambda)=\lambda /(1+\lambda)$, independent of the coupling function $w$. In this case the only root of $\varepsilon(\lambda)$ is $\lambda=0$.

It can be shown that the essential spectrum associated with the stability of $q$ lies strictly in the left half of the complex plane [32] and, combining this with the result immediately above, we see that stationary front solutions of (24) are linearly stable.

\subsection{Results}

We now put together the results from Sections 5.2 and 5.3 by plotting the solution curves, the speed of travelling fronts and the Hamiltonian at the nonzero fixed point $\hat{\mathbf{Z}}$ as functions of $b$ in Fig. 14. The breaks in the solution curves occur for the same two values of $b$ for which stationary fronts (heteroclinic connections) exist and for the parameter values at which the Hamiltonian of the nonzero fixed point is equal to zero. So - for this value of $\theta-$ at two values of $b$ a global bifurcation creates a heteroclinic connection at the breakpoints of the solution curves, destroying the homoclinic orbits in the region of the break.

\section{Discussion}

We have examined the unexpected disappearance of stable homoclinic orbits in certain regions of parameter space in a neural field model with one spatial dimension, using the decaying oscillatory coupling function and smooth firing rate function studied previously in $[7,21]$. For a particular firing threshold, we have shown that the solution curve of stable homoclinic orbits to the fixed point at the origin breaks when the firing rate function is sufficiently steep, destroying stable homoclinic
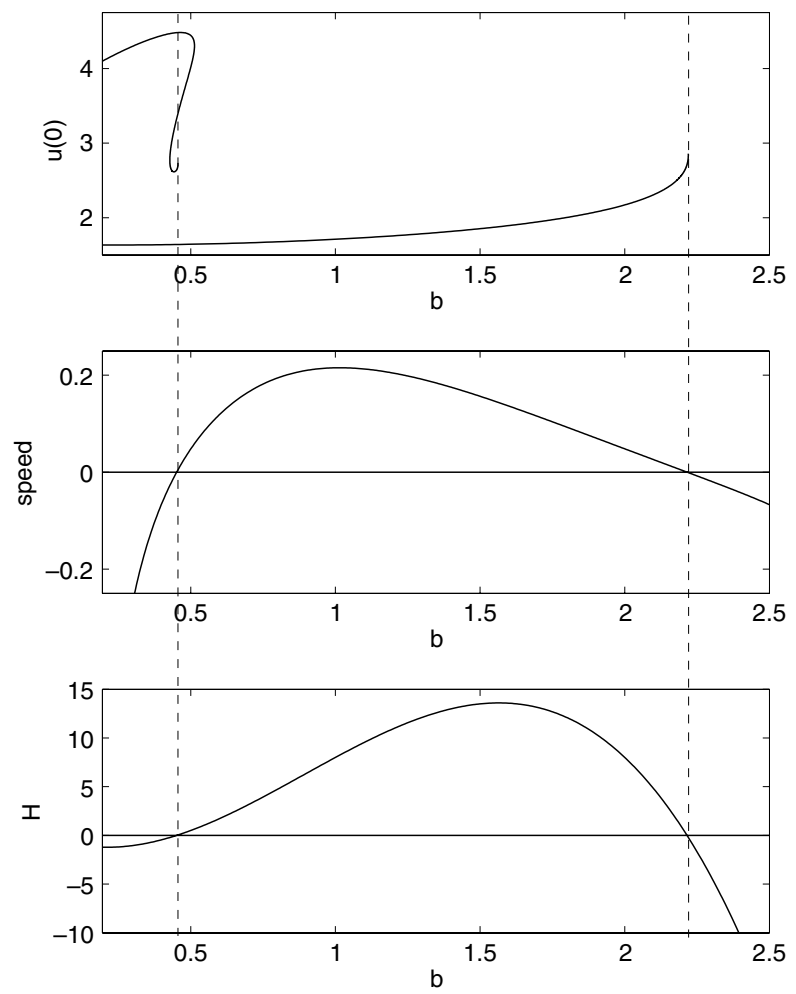

Fig. 14. Top: Single bump solution curves of (1) and (3) with $f(u)=2 \Theta(u-1.5)$. Middle: Speed of travelling fronts, $c$, in (26) as a function of $b$. Bottom: Hamiltonian at the nonzero fixed point $\hat{\mathbf{Z}}$. The breaks in the solution curves occur for the same values of $b$ for which both stationary fronts exist and the Hamiltonian of $\hat{\mathbf{Z}}$ is zero.

orbits in a region of parameter space. The sudden break in the solution curve is unexpected and unexplained. Through the use of Fourier transforms, the equation satisfied by the steady state was written as a fourth-order reversible Hamiltonian system [7] and the properties of such systems were exploited to explain the phenomenon.

Using a two-dimensional mapping, we have reduced the problem of finding homoclinic orbits to finding the zeroes of a real scalar function and discovered that a small separate solution curve exists when the firing rate function is sufficiently steep. Standard continuation techniques were insufficient to discover this curve, as we had no starting solution on that particular branch of solutions. We have found that, as the firing function steepens, this small curve merges with the main solution curve, causing it to break and the gap to widen. Within the region of the break, no stable homoclinic orbits to the fixed point at the origin exist, and a codimension-one bifurcation occurs at the breakpoints in the solution curve, where the stable and unstable manifolds of the fixed point at the origin and a nonzero fixed point collide. There appear to be spirals at the terminating ends of the solution curve.

By restricting the firing rate function to be a multiple of the Heaviside step function, we have used analytical techniques to show that the firing threshold has to be at an appropriate level for the solution curves to break and that stationary fronts (heteroclinic connections) exist at the breakpoints. Although the global bifurcation studied in this paper has not been previously seen in a neural field model, the bifurcation is very similar to the codimension-two bifurcation seen in two-dimensional parameter space in the Lorenz equations (a $T$-point) [29].

The model (1)-(3), and other similar models [6,10,24,33], have been used to investigate working memory, as stable single-bump solutions are thought to be the analogue of short-term memory. We have demonstrated here how a specific model can lose stable 
single-bump solutions as parameters are varied. This gives insight into some of the features that such a model must have in order for it to be able to support such solutions. It is interesting to note that one of the solutions seen in the break in Fig. 3 is a travelling front, similar to those observed in slice experiments [35,36].

In general, we want a qualitative model to be robust to small changes in parameters. However, the phenomena we have seen occurs in a certain region of parameter space that has not been previously studied, and the rich behaviour displayed is of interest in its own right. Further areas of study arise out of the work presented here. We do not yet know what causes the isola to spring into life as the firing rate function steepens nor do we fully understand the apparent spirals occurring at the terminating ends of the solution curves. As stationary fronts occur at the breakpoints in the solution curves, we also note an exploration of travelling fronts in the region of discontinuity of the solution curves, as shown in Fig. 4, as a topic for further study.

\section{Acknowledgment}

The first author thanks E. Knobloch for useful discussions.

\section{References}

[1] A.R. Champneys, Homoclinic orbits in reversible systems and their applications in mechanics, fluids and optics, Physica D 112 (1998) 158-186.

[2] G.W. Hunt, M.A. Peletier, A.R. Champneys, P.D. Woods, M. Ahmer Wadee, C.J. Budd, G.J. Lord, Cellular buckling in long structures, Nonlinear Dyn. 21 (2000) 3-29.

[3] M. Tlidi, P. Mandel, R. Lefever, Localized structures and localized patterns in optical bistability, Phys. Rev. Lett. 73 (1994) 640-643.

[4] P.B. Umbanhowar, F. Melo, H.L. Swinney, Localized excitations in a vertically vibrated granular layer, Nature 382 (1996) 793.

[5] J.H.P. Dawes, Localized convection cells in the presence of a vertical magnetic field, J. Fluid Mech. 570 (2007) 385-406.

[6] Y. Guo, C. Chow, Existence and stability of standing pulses in neural networks: I. Existence, SIAM J. Appl. Dyn. Syst. 4 (2005) 217-248.

[7] C.R. Laing, W.C. Troy, B. Gutkin, G.B. Ermentrout, Multiple bumps in a neuronal model of working memory, SIAM J. Appl. Math. 63 (1) (2002) 62-97.

[8] S. Amari, Dynamics of pattern formation in lateral-inhibition type neural fields, Biol. Cybern. 27 (2) (1977) 77-87.

[9] G.B. Ermentrout, Neural networks as spatio-temporal pattern-forming systems, Rep. Prog. Phys. 61 (1998) 353-430.

[10] D.J. Pinto, G.B. Ermentrout, Spatially structured activity in synaptically coupled neuronal networks: II. Lateral inhibition and standing pulses, SIAM J. Appl. Math. 62 (1) (2001) 226-243.

[11] J. Burke, E. Knobloch, Localized states in the generalised Swift-Hohenberg equation, Phys. Rev. E 73 (2006) 056211.

[12] J. Burke, E. Knobloch, Homoclinic snaking: Structure and stability, Chaos 17 (2007) 037102.
[13] J. Burke, E. Knobloch, Snakes and ladders: Localized states in the Swift-Hohenberg equation, Phys. Lett. A 360 (2007) 681-688.

[14] E. Knobloch, Spatially localized structures in dissipative systems: Open problems, Nonlinearity 21 (2008) T45-T60.

[15] L.A. Peletier, W.C. Troy, Spatial Patterns: Higher Order Models in Physics and Mechanics, Birkhauser, Boston, 2001.

[16] D.J.B Lloyd, B. Sandstede, D. Avitabile, A.R. Champneys, Localized hexagon patterns of the planar Swift-Hohenberg equation, SIAM J. Appl. Dyn. Syst. 7 (3) (2008) 1049-1100.

[17] C.R. Laing, W.C. Troy, PDE methods for nonlocal models, SIAM J. Appl. Dyn. Syst. 2 (3) (2003) 487-516.

[18] B.S. Gutkin, G.B. Ermentrout, J. O’Sullivan, Layer 3 patchy recurrent excitatory connections may determine the spatial organization of sustained activity in the primate prefrontal cortex, Neurocomputing 32-33 (2000) 391-400.

[19] J.B. Levitt, D.A. Lewis, T. Yoshioka, J.S. Lund, Topography of pyramidal neuron intrinsic connections in macaque monkey prefrontal cortex (areas 9 and 46), J. Comput. Neuro. 338 (1993) 360-376.

[20] D.A. Lewis, S.A. Anderson, The functional architecture of the prefrontal cortex and schizophrenia, Psych. Med. 25 (1995) 887-894.

[21] A.J. Elvin, Pattern formation in a neural field model. Ph.D. Thesis, Massey University, 2008. http://hdl.handle.net/10179/717.

[22] A.J. Elvin, C.R. Laing, M.G. Roberts, Transient Turing patterns in a neural field model, Phys. Rev. E 79 (2009) 011911.

[23] S. Coombes, G.J. Lord, M.R. Owen, Waves and bumps in neuronal networks with axo-dendritic synaptic interactions, Physica D 178 (3-4) (2003) 219-241.

[24] S. Coombes, Waves, bumps, and patterns in neural field theories, Biol. Cybern. 93 (2) (2005) 91-108.

[25] S. Coombes, N.A. Venkov, L. Shiau, I. Bojak, D.T.J. Liley, C.R. Laing, Modeling electrocortical activity through improved local approximations of integral neural field equations, Phys. Rev. E 76 (5) (2007) 51901.

[26] V.K. Jirsa, H. Haken, Field theory of electromagnetic brain activity, Phys. Rev. Lett. 77 (5) (1996) 960-963.

[27] E.J. Doedel, R.C. Paffenroth, A.R. Champneys, T.F. Fairgrieve, Y.A. Kuznetsov, B.E. Oldeman, B. Sandstede, X. Wang, AUTO 2000: Continuation and bifurcation software for ordinary differential equations (with HomCont), 2000.

[28] P.G. Drazin, Nonlinear Systems, Cambridge University Press, 2002.

[29] P. Glendinning, C. Sparrow, T-points: A codimension two heteroclinic bifurcation, J. Stat. Phys. 43 (3-4) (1986).

[30] S. Coombes, M.R. Owen, Bumps, breathers, and waves in a neural network with spike frequency adaptation, Phys. Rev. Lett. 94 (2005).

[31] Z.P. Kilpatrick, P.C. Bressloff, Effects of synaptic depression and adaptation on spatiotemporal dynamics of an excitatory neuronal network, Physica D (2009), in press (doi:10.1016/j.physd.2009.06.003).

[32] S. Coombes, M.R. Owen, Evans functions for integral neural field equations with Heaviside firing rate function, SIAM J. Appl. Dyn. Syst. 3 (4) (2004) 574-600.

[33] C. Laing, S. Coombes, The importance of different timings of excitatory and inhibitory pathways in neural field models, Network: Comp. Neural Sys. 17 (2) (2006) 151-172.

[34] M.R. Owen, C.R. Laing, S. Coombes, Bumps, rings, and spots in a twodimensional neural field: Splitting and rotational instabilities, New J. Phys. 9 (2007) 378

[35] R.D. Traub, J.G. Jefferys, R. Miles, Analysis of the propagation of disinhibitioninduced after-discharges along the guinea-pig hippocampal slice in vitro, J. Physiol. 472 (1993) 267-287.

[36] J.-Y. Wu, X. Huang, C. Zhang, Propagating waves of activity in the neocortex: What they are, what they do, The Neuroscientist 14 (2008) 487-502. 